\title{
Application of Statistical Image Analysis in Quantification of Neutron Radiography Images of Drying
}

\author{
I.M. Fiją-KirejCzyK ${ }^{a, *}$, J.J. MilczAreK ${ }^{a}$, J. ŻoŁĄDeK-NowaK ${ }^{a}$, F.C. De Beer ${ }^{b}$, \\ M.J. RADEBE ${ }^{b}$ AND G. NOTHNAGEL ${ }^{b}$ \\ ${ }^{a}$ National Centre for Nuclear Research (NCBJ), A. Sołtana 7, 05-400 Otwock-Świerk, Poland \\ ${ }^{b}$ Radiation Science, Necsa, Church Street West ext., Pelindaba, Pretoria, 0001, South Africa
}

\begin{abstract}
The statistical approach to the thermal neutron radiography picture analysis is applied to evaluation of the experimental results obtained for drying of rectangular and cylindrical samples of granulated and rigid porous materials. We have shown that the time dependence of the standard deviation of image brightness reflects the appearance and motion of the drying front observed during second period of the drying process. The results are discussed within a simple two-region model of the drying sample image.
\end{abstract}

PACS: 87.59.bf, 07.05.Pj, 61.05.Tv, 81.05.Rm, 47.56. $+\mathrm{r}, 47.55 . \mathrm{nb}$

\section{Introduction}

Although drying has been studied for almost a century, the role of various processes of mass and heat transfer within a drying systems is still discussed [1-8] and new experimental data on drying are welcomed and thoroughly analyzed. It is considered that the simplest drying systems are those formed of capillary porous media. For these systems the traditional division of drying into three main periods, named constant rate (CRP), first and second falling rate periods (FRP) according to the time behavior of rate of mass decrease of drying specimen remains valid [1-5]. The CRP is determined by the evaporation rate from the sample surface and efficiency of capillary forces driving the water to the sample surface from the water filled pores. In the first FRP the flow of water films covering the surfaces of partially filled with water capillary channels is important. During the second FRP the moisture is transported from the moderately wet central core to the sample surface by vapor diffusion through capillaries [1-3]. At this stage the rather sharp boarder surface called the submerged or receding (from sample surface) drying front limiting the wet core is formed [3-9]. One should note that during last twenty years a relevant progress in the investigations of drying has been brought about by application of new imaging techniques like NMR [10], X-ray [11, 12] and thermal neutron imaging [13-23], which permit detailed observation of spatial moisture distribution in the porous sample.

Recent application of thermal neutron radiography (NR) in the observations of drying processes of capillary-porous media has created new possibilities of determination of moisture distribution inside the body of drying

\footnotetext{
* corresponding author; e-mail:

Izabela.Fijal-Kirejczyk@ncbj.gov.pl
}

specimen. In particular, the drying front $[9,17,18,21]$ and its motion across the sample volume have been visualized and quantified from the recorded sequences of neutron radiographs. The aim of present work has been the statistical description of the neutron radiographs corresponding to the main phases of drying process in capillary-porous samples of different shape.

The merit of neutron imaging in revealing the presence of water in porous media stems from very strong scattering of thermal neutrons by hydrogen nuclei, which removes neutrons from the incident beam producing dark regions in the images of water containing samples [13-16]. Due to small attenuation of neutrons by most materials, the NR provides the possibility of revealing the spatial distribution of water in the sample body [1, 5, 13-22].

The radiography studies of processes consist in quantification of the images not only with pure visual inspection of noise corrected and contrast enhanced radiographs. Usually, in order to find some emerging features in the samples studied the pictures are analyzed for changes in brightness as well as distribution of grey levels among pixels $[18,21]$. In this work we suggest the additional use of the brightness standard deviation as a measure of picture diversification that delineates patterns emerging during the observed process. In the analysis the digital representation of the picture of the sample is considered as the stochastic matrix whose elements take values from the set of the encoded grey levels.

The statistical approach to the neutron radiographs analysis is here demonstrated with the experimental results obtained for drying of rectangular and cylindrical samples of granulated and rigid porous materials.

\section{Experiments}

The experiments were carried out at the neutron radiography station (NGRS) at the MARIA nuclear research reactor of NCBJ [19-23]. The drying process was 
observed with on-line registration of the sample mass and temperature as well as sequences of neutron radiographs for the whole process. The experimental system was essentially the same for drying of mortar rectangular block and quartz sand cylinders. The sample was dried in the drying tunnel with $60^{\circ} \mathrm{C}$ hot air stream flowing from above at the rate of $0.03 \mathrm{~m}^{3} \mathrm{~s}^{-1}$ (stream velocity $\left.\approx 3 \mathrm{~m} \mathrm{~s}^{-1}\right)$. The sample's temperature and mass were recorded every $10 \mathrm{~s}$ and the neutron radiographs every $2 \mathrm{~s}$.

Two samples made of different porous materials were investigated. The first sample made of aged special mortar was a rectangular block of $48 \mathrm{~mm} \times 52 \mathrm{~mm} \times 14 \mathrm{~mm}$, which was saturated with water by immersion for $48 \mathrm{~h}$. With the effective porosity of the mortar of $\approx 60 \%$, the initial mass content of water in the sample was $\approx 26 \%$. The second sample was a cylinder of $20 \mathrm{~mm}$ diameter and $30 \mathrm{~mm}$ height prepared from quartz sand. This sample was produced by extrusion and immediately placed inside a dryer tunnel, the initial mass content of water was $\approx 16 \%$ of weight and the effective porosity $\approx 40 \%$.

The samples were investigated separately in the drying tunnel at the NGRS [19-21]. Each of them with appropriate cover on its top was placed on aluminum support rod with its lower end resting on the electronic balance tray. The balance provided standard accuracy of $0.1 \mathrm{~g}$ for items of mass less than $500 \mathrm{~g}$. The sample support incorporated the K-type steel sheathed thermocouple of $0.5 \mathrm{~mm}$ diameter. The thermocouple penetrated the sample vertically from its lower end into the bulk with thermocouple head placed at the distance of $\approx 15 \mathrm{~mm}$ from the sample bottom.

The NR comprises the neutron beam collimators, fluorescent screen $\left(250 \times 250 \mathrm{~mm}^{2}\right)$, mirror, optical zoom lenses and CCD camera. The high sensitivity CCD camera, Hamamatsu ORCA-ER $(1280 \times 1024$ pixels, 12 bits $)$, is used in the system. The collimating $L / D$ ratio during reported experiments was $\approx 165$ and the exposure time was $1.6 \mathrm{~s}$. The projection ratio provided by the optical system was $154 \mu \mathrm{m} /$ pixel. The sample was placed at the distance of $\approx 65 \mathrm{~mm}$ from the converter screen. The image analysis was performed with the LUCIA ${ }^{\mathrm{TM}} 4.60$, SigmaScan Pro 5.0.0 and ImageJ $1.43 \mathrm{u}$ software packages. Before image analysis pre-processing procedures including the correction of pixel brightness for the black current, normalization for neutron beam flux fluctuations, as well as the median filtering was applied.

\section{Results}

The time dependence of the brightness of the neutron image of the sample corresponds to the changes in its water content. During the first period of the process the samples were uniformly saturated with water. However, in the second period of drying the darker central region emerged (Figs. 1 and 2). That darker inner region was separated from the lighter outer region by a discernible boundary corresponding to the drying front. One should remember that the drying front is a surface boundary of a wet core region in the sample's volume. The registered neutron radiographs reveal the shrinking of the inner dark region towards the centre of the sample. This region represents the sample's inner wet region, which contains the amount of water detectable by neutrons. Due to significant difference in brightness of the observed two regions the emergence of this pattern increases considerably the standard deviation of the image brightness. Further on, the receding front disappears leaving more or less uniform image. In effect the maximum is observed in the time dependence of the standard deviation of image brightness. At the end of the process the sample image was almost uniform for both rectangular and cylindrical samples.

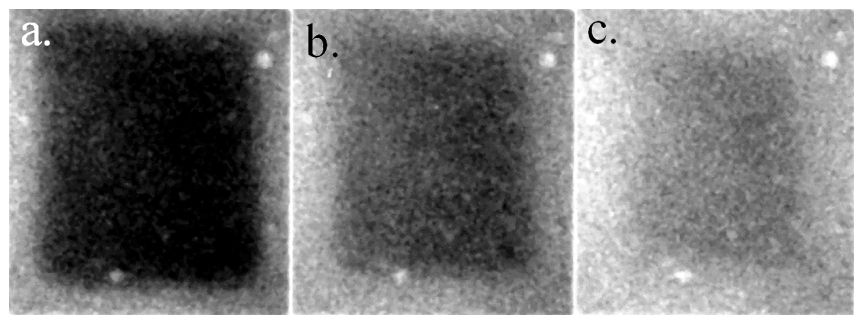

Fig. 1. The time evolution of the rectangular mortar sample image during drying. Parts (a)-(c) correspond to the times 6920,8400 , and $9610 \mathrm{~s}$, respectively.

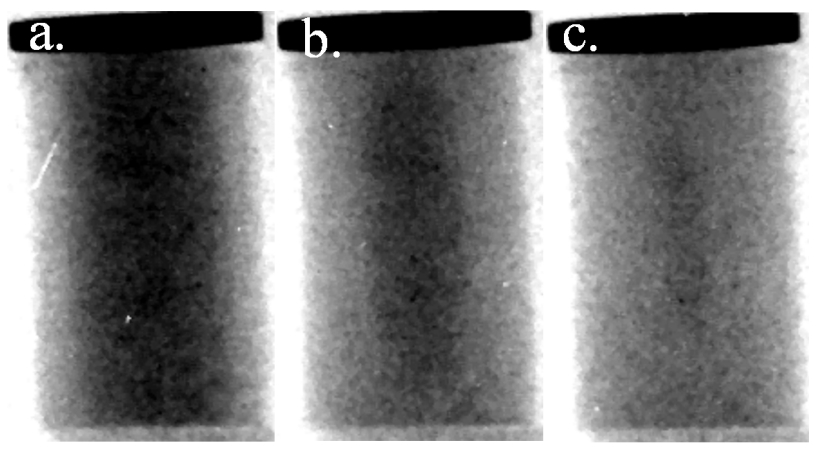

Fig. 2. The time evolution of the cylindrical quartz sand sample image during drying. Parts (a)-(c) correspond to the times 2100,2500 , and $2840 \mathrm{~s}$, respectively.

In the stochastic analysis we treat the sample image as a stochastic matrix with elements corresponding to image pixels and given by brightness of the pixels. The sample image was cropped from the all sequences of the recorded neutron radiographs with a simple procedure. In particular, the image of mortar sample was a matrix with $310 \times 340$ elements, and for sand quartz sample it was $110 \times 200$ matrix of integers from 0 to 255 (in case of 8 bit encoding of the grey level). For each sample image the brightness histogram $H(b)$ was determined. Since in case of discrete stochastic variable $b$ the $H(b)$ is the number of pixels with given brightness $b$ it was easily calculated with ImageJ software package. The time evolution 


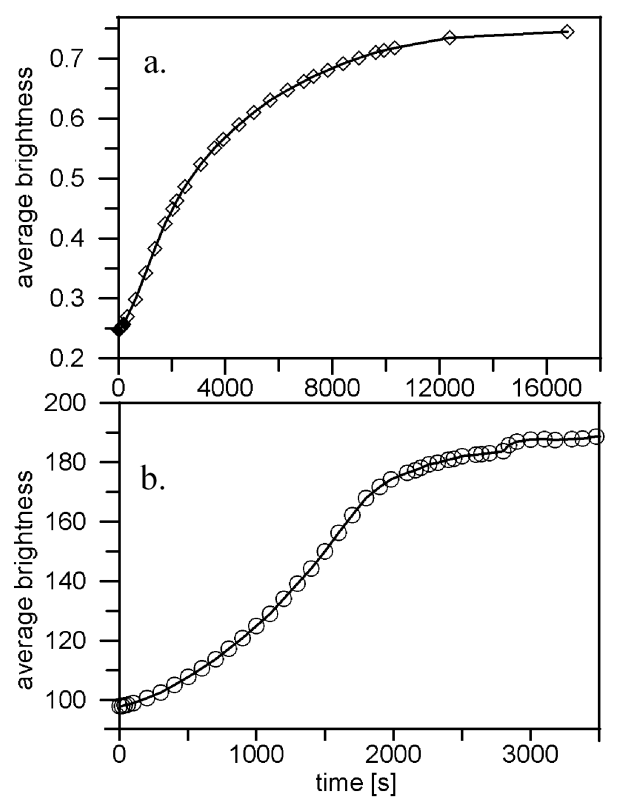

Fig. 3. The time variation of sample image brightness for mortar (a) and quartz sand (b) samples.

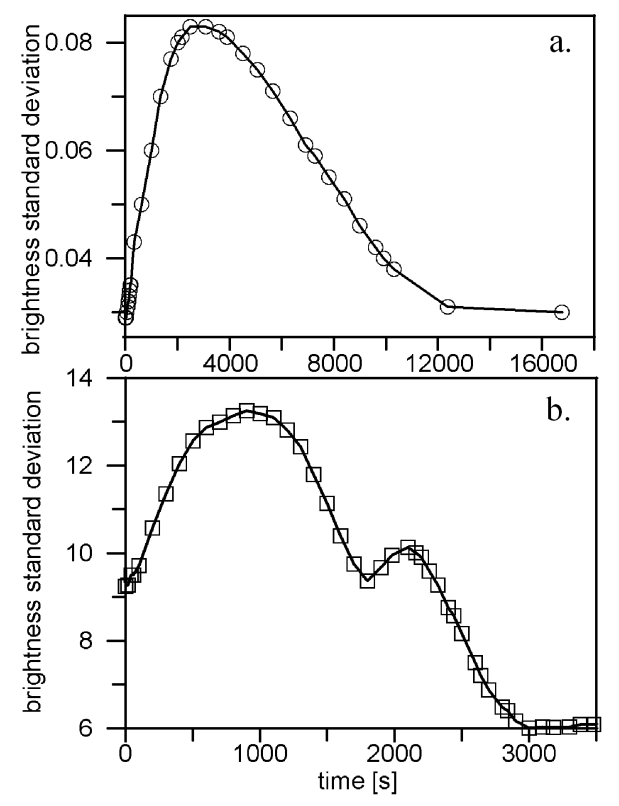

Fig. 4. The time variation of the standard deviation of the sample image brightness for mortar (a) and quartz sand (b) samples.

of the image histogram revealed very profound changes in its shape during drying. At the beginning $H(b)$ consisted of a single peak corresponding to uniform image with dark grey pixels. Then a broadening and shift of the histogram to the larger $b$ region was observed. With determined histograms the moments of brightness can be calculated according to usual definitions

$$
\begin{aligned}
& \left\langle b^{n}\right\rangle=\frac{\int b^{n} H(b) \mathrm{d} b}{\int H(b) \mathrm{d} b}, \\
& \Delta=\left(\left\langle b^{2}\right\rangle-\langle b\rangle^{2}\right)^{1 / 2} .
\end{aligned}
$$

The average brightness $\langle b\rangle$ of the sample image increased very steeply with time during the first period of drying and levelled off rather smoothly at the advanced period for both samples studied (Fig. 3). The time dependence of the standard deviation $\Delta$ is more complex with one maximum observed for the rectangular sample (Fig. 4a) and two maxima for the cylindrical one (Fig. 4b).

\section{Discussion}

It is obvious that the increase in average brightness of the sample's image is due to main feature of the drying i.e. the decreasing sample's average water content. The observations of the maxima in the plots of the brightness standard deviation can be attributed to the development of the outer almost dry layer in the sample surrounding the inner wet core. The time evolution of two image regions of different brightness corresponds to the kinetics of the dry and wet parts of the sample. The appearance of the dry outer layer produces a light frame surrounding the darker central region in neutron radiographs. The border between those regions is the image of the receding drying front separating the wet core from the dry outer region. In effect the diversity in the gray level distribution in the sample image appears enhancing the standard deviation of brightness. As the thickness of that lighter frame grows, the standard deviation initially increases and attains a maximum when the areas of both regions are equal. Further on, the lighter outer region overwhelms the dark core producing more even distribution of gray levels and reducing the brightness standard deviation. This reasoning can be explained more definitely using a very simple model of the image consisting of two regions, a dark one denoted as $W$, corresponding to wet part of the sample, and a light denoted as $D$ due to dry part.

Denoting by $p_{W}$ and $p_{D}=1-p_{W}$ the fractions of the sample image areas, and by $b_{W}$ and $b_{D}$ the corresponding brightness of the wet and dry regions, respectively, one gets for the average brightness

$$
\langle b\rangle=b_{W}+\left(b_{D}-b_{W}\right) p_{D},
$$

and for the square of the standard deviation

$$
\Delta^{2}=p_{D}\left(1-p_{D}\right)\left(b_{D}-b_{W}\right)^{2} \text {. }
$$

It is obvious that the standard deviation attains here only one maximum for $p_{D}=1 / 2$ which corresponds to the equal fractional areas of the images of the wet and dry regions.

In order to explain the two maxima found in the $\Delta^{2}$ vs. $t$ plot for the cylindrical sample one should consider the non-uniformity of the homogeneous cylinder image due to the different neutron path for the rays transmitted through the outer and central regions of the cylinder. 
The scattered neutrons smooth the distribution of the brightness over the cylinder image [15, 16, 18, 19-21].

We have proved recently [21] that during the first drying period the saturation with water is uniform across the sample. With decreasing water content the scattered neutrons component becomes less important and in effect at the end of the first drying period stronger diversification in the gray level distribution is produced yielding initial increase observed in the standard deviation versus time plot. Then the decreasing but still homogeneous saturation of the sample with water produces smaller attenuation of neutrons that reduces the differences in brightness between outer and central parts of the cylinder image.

In effect the first maximum in the standard deviation is produced (Fig. 4b). This reasoning can be expressed quantitatively with results of the analysis of our data obtained previously for drying of similar quartz cylinder [21]. The brightness profiles $b(x)$ along the line perpendicular to the sample axis at its middle were fitted with two components, $a(x)$ and $s(x)$, representing the attenuation and the scattering of the parallel neutron beam, respectively.

Almost linear decrease with time was found for neutron macroscopic cross-section and the scattering neutron component amplitude by fitting the neutron image brightness profiles. Using these results the brightness moments for line profiles were calculated by numerical integration of attenuated and scattered beam components [21] over the sample image width $(2 R)$ :

$$
\left\langle b^{n}\right\rangle=(2 R)^{-1} \int_{-R}^{R} b^{n}(x) \mathrm{d} x .
$$

The calculation results reveal a small initial increase of the brightness standard deviation with and its subsequent sharp decrease when the scattering component disappeared (Fig. 5). The flatness of that maximum is due to the one-dimensionality of the model.

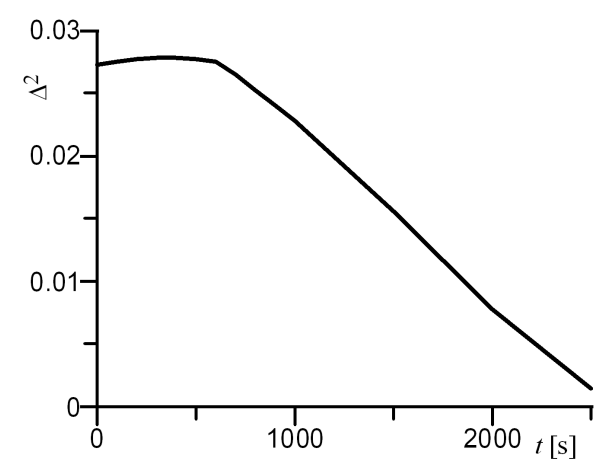

Fig. 5. The results of the numerical calculations of brightness standard deviation on central line perpendicular to the axis of the drying cylinder uniformly saturated with water.

The second maximum in $\Delta^{2}$ vs. $t$ plot is exact analogue of the effect observed in the rectangular sample.
At the beginning of the second period the dry outer region begins to develop yielding two distinct regions in the cylindrical sample image as it was found for the rectangular sample. As the light frame becomes wider the standard deviation of brightness initially increases to reach a maximum and then decreases as the partial area corresponding to the wet region image diminishes.

We should note that the standard deviation given by Eq. (4), depends on the area of the inner core region image provided as well as on the difference in brightness of the two distinct regions observed. In effect the determination of the rate of the drying front motion by the analysis of the time variation of brightness standard deviation is difficult. We believe that the direct determination of the front position from neutron radiographs provides more reliable data for analysis of the drying front kinetics during the last stage of the process.

\section{Conclusions}

We have shown that the drying front occurring in the second drying period can be visualized with thermal neutron radiography for samples of different shape made of different materials. We proposed and applied the statistical approach of picture analysis to prove that the standard deviation of the sample image brightness is appropriate measure of the inhomogeneities emerging in the drying sample. In particular the existence and time evolution of the inner wet core was analyzed. A simple two-region model was presented for qualitative explanation of single maximum in standard deviation dependence on time during development and motion of the drying front across the rectangular sample. The two maxima in brightness standard deviation on time dependence were explained in terms of the vanishing scattered neutrons contribution to the image brightness profiles and subsequent emergence of two distinct regions.

\section{Acknowledgments}

This work has been supported by Ministry of Science and Higher Education of Republic of Poland and National Science Foundation of Republic of South Africa within the "Neutron Radiography/Tomography of Porous Media" project No. 7963/R10/R11 performed according to the Scientific and Technological Co-operation Agreement between Poland and South Africa.

\section{References}

[1] J.M. Coulson, J.F. Richardson, Chemical Engineering, Vol. 2, Pergamon Press, London 1956.

[2] D.A. Brosnan, G.C. Robinson, Introduction to Drying of Ceramics, American Ceramic Society, Westerville 2003.

[3] A.V. Luikov, Int. J. Heat Mass. Transfer. 18, 1 (1975).

[4] J. van Brakel, in: Advances in Drying, Ed. A.S. Mujumdar, Hemisphere, New York 1980, p. 217. 
[5] E.U. Schlünder, Drying Technol. 22, 1517 (2004).

[6] I.N. Tsimpanogiannis, Y.C. Yortsos, S. Poulou, N. Kanellopoulos, A.K. Stubos, Phys. Rev. E 59, 4353 (1999).

[7] P. Coussot, Eur. Phys. J. B 15, 557 (2000).

[8] P. Lehmann, S. Assouline, D. Or, Phys. Rev. E 77, 56309 (2008).

[9] P. Lehmann, D. Or, Phys. Rev. E 80, 046318 (2009).

[10] I.V. Koptyug, Prog. Nucl. Magn. Reson. Spectrosc., in press, 2011, doi: 10.1016/j.pnmrs.2011.12.01.

[11] M. Kohout, Z. Grof, F. Stepanek, J. Colloids Interface Sci. 299, 342 (2006).

[12] D. Wildenschild, J.W. Hopmans, C.M.P. Vaz, M.L. Rivers, D. Rikard, B.S.B. Christensen, J. Hydrol. 267, 285 (2002).

[13] Practical Neutron Radiography, Ed. J.C. Domanus, Kluwer Academic Pub., Dordrecht 1992.

[14] Neutron Imaging and Applications, Eds. I.S. Anderson, R.L. McGreevy, H.Z. Bilheux, Springer, Berlin 2009 .

[15] M.R. Deinert, J.-Y. Parlange, T. Steenhuis, J. Throop, K. Ünlü, K.B. Cady, J. Hydrol. 290, 192 (2004).
[16] E.H. Lehmann, P. Vontobel, N. Kardjilov, Appl. Radiat. Isot. 61, 503 (2004).

[17] F.C. de Beer, W.J. Strydom, E.J. Griesel, Appl. Radiat. Isot. 61, 617 (2004).

[18] N. Shokri, P. Lehmann, P. Vontobel, D. Or, Water Resour. Res. 44, W06418 (2008).

[19] I. Fijał-Kirejczyk, J.J. Milczarek, J. Banaszak, A. Trzciński, J. Żołądek, Nukleonika 54, 123 (2009).

[20] I. Fijal-Kirejczyk, J.J. Milczarek, J. Banaszak, J. Żołądek, A. Trzciński, Def. Diff. Forum 297-301, 508 (2010).

[21] I.M. Fijał-Kirejczyk, J.J. Milczarek, J. Żołądek-Nowak, Nucl. Instrum. Methods Phys. Res. A 651, 201 (2011).

[22] J.J. Milczarek, A. Czachor, A. Abd, Z. El-Ghany, Wiśniewski, Nucl. Instrum. Methods Phys. Res. A 542, 232 (2005).

[23] J. Żołądek, J.J. Milczarek, I. Fijał-Kirejczyk, Nukleonika 53, S113 (2008). 